\title{
Growth differentiation factor-15 and the association between type 2 diabetes and liver fibrosis in NAFLD
}

\author{
Josh Bilson (D) ${ }^{1,2}$, Eleonora Scorletti ${ }^{1,2,3}$, Laure B. Bindels (D) $^{4}$, Paul R. Afolabi ${ }^{1,2}$, Giovanni Targher (D) ${ }^{5}$, Philip C. Calder ${ }^{1,2,6}$,

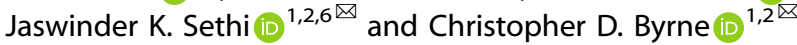

(c) The Author(s) 2021

BACKGROUND: Type 2 diabetes mellitus (T2DM) is a strong risk factor for liver fibrosis in non-alcoholic fatty liver disease (NAFLD). It remains uncertain why T2DM increases the risk of liver fibrosis. It has been suggested that growth differentiation factor-15 (GDF-15) concentrations increase the risk of liver fibrosis. We aimed to investigate (a) whether GDF-15 concentrations were associated with liver fibrosis and involved in the relationship between T2DM and liver fibrosis and (b) what factors linked with T2DM are associated with increased GDF-15 concentrations.

METHODS: Ninety-nine patients with NAFLD (61\% men, 42.4\% T2DM) were studied. Serum GDF-15 concentrations were measured by electro-chemiluminescence immunoassay. Vibration-controlled transient elastography (VCTE)-validated thresholds were used to assess liver fibrosis. Regression modelling, receiver operator characteristic curve analysis and Sobel test statistics were used to test associations, risk predictors and the involvement of GDF-15 in the relationship between T2DM and liver fibrosis, respectively. RESULTS: Patients with NAFLD and T2DM $(n=42)$ had higher serum GDF-15 concentrations [mean (SD): 1271.0 (902.1) vs. 640.3 (332.5) $\mathrm{pg} / \mathrm{ml}, p<0.0001$ ], and a higher proportion had VCTE assessed $\geq \mathrm{F} 2$ fibrosis $(48.8$ vs. $23.2 \%, p=0.01)$ than those without T2DM. GDF-15 was independently associated with liver fibrosis $(p=0.001)$, and GDF-15 was the most important single factor predicting $\geq \mathrm{F} 2$ or $\geq \mathrm{F} 3$ fibrosis ( $\geq \mathrm{F} 2$ fibrosis AUROC $0.75,(95 \% \mathrm{Cl} 0.63-0.86), p<0.001$, with sensitivity, specificity, positive predictive (PPV) and negative predictive (NPV) values of $56.3 \%, 86.9 \%, 69.2 \%$ and $79.1 \%$, respectively). GDF-15 was involved in the association between T2DM and $\geq F 2$ fibrosis (Sobel test statistic 2.90, $p=0.004$ ). Other factors associated with T2DM explained 60\% of the variance in GDF-15 concentrations $(p<0.0001)$. HbA1c concentrations alone explained $30 \%$ of the variance $(p<0.0001)$. CONCLUSIONS: GDF-15 concentrations are a predictor of liver fibrosis and potentially involved in the association between T2DM and liver fibrosis in NAFLD. HbA1c concentrations explain a large proportion of the variance in GDF-15 concentrations.

Nutrition and Diabetes (2021)11:32; https://doi.org/10.1038/s41387-021-00170-3

\section{INTRODUCTION}

Non-alcoholic liver disease (NAFLD) is a 'multisystem' disease that increases the risk of type 2 diabetes mellitus (T2DM), cardiovascular disease (CVD) [1], and chronic kidney disease (CKD) [2]. Bidirectional relationships exist between NAFLD and T2DM and not only is NAFLD an independent risk factor for incident T2DM, but when both diseases co-exist, T2DM increases the risk of faster progression of NAFLD to advanced fibrosis, cirrhosis and hepatocellular carcinoma [2-4]. However, the factors involved in the association between T2DM and increased risk of liver fibrosis in patients with NAFLD are not fully understood.

Growth differentiation factor-15 (GDF-15), also known as macrophage inhibitory cytokine (MIC)-1, is a stress-inducible cytokine that can be ubiquitously expressed [5]. Circulating GDF15 concentrations are increased in patients with T2DM [6, 7], and are separately reported to associate with obesity [8], liver disease severity [9, 10], CVD [11] and CKD [12]. A recent multicentre transcriptomic study demonstrated that hepatic GDF-15 expression was positively associated with NAFLD severity and GDF-15 expression was significantly higher in patients with advanced liver fibrosis [10]. In support of these findings, a previous study measured serum GDF-15 concentrations in patients (in an Asian population) with both T2DM and NAFLD and showed that GDF-15 concentrations were higher in those with T2DM and advanced liver fibrosis [13]. Furthermore, growing evidence suggests that GDF-15 may have pro-fibrogenic effects within the liver and other tissues [13-15]. Taken together, these studies suggest that increased GDF-15 concentrations may increase the risk of liver fibrosis. However, it is not known whether circulating GDF-15 concentrations are potentially involved in the known relationship between T2DM and liver fibrosis in patients with NAFLD.

Additional factors associated with T2DM have also been proposed to explain why T2DM is a risk factor for liver fibrosis. These include insulin resistance, altered adipokine concentrations

\footnotetext{
${ }^{1}$ Human Development and Health, Faculty of Medicine, University of Southampton, Southampton, UK. ${ }^{2}$ National Institute for Health Research Southampton Biomedical Research Centre, University of Southampton and University Hospital Southampton National Health Service Foundation Trust, Southampton, UK. ${ }^{3}$ Division of Gastroenterology, Department of Medicine, Perelman School of Medicine, University of Pennsylvania, Philadelphia, PA 19104, USA. ${ }^{4}$ Metabolism and Nutrition Research Group, Louvain Drug Research Institute, Université Catholique de Louvain, Brussels, Belgium. ${ }^{5}$ Section of Endocrinology, Diabetes and Metabolism, Department of Medicine, University and Azienda Ospedaliera Universitaria Integrata of Verona, Verona, Italy. ${ }^{6}$ Institute for Life Sciences, University of Southampton, Southampton, UK. ${ }^{凶}$ email: J.Sethi@soton.ac.uk; C.D.Byrne@soton.ac.uk
} 
[16] and altered gut microbiota composition [17]. Moreover, it has also been suggested that oral hypoglycaemic agents, such as metformin, can affect circulating GDF-15 concentrations [18, 19]. Whether these factors explain the increase in GDF-15 concentration in patients with T2DM and NAFLD remains uncertain. Therefore we aimed to test (a) whether GDF-15 concentrations were a predictor of liver fibrosis and potentially involved in the association between T2DM and liver fibrosis and (b) what factors linked with T2DM are independently associated with, and explain the variance in GDF-15 concentrations, in patients with NAFLD.

\section{MATERIALS AND METHODS}

A total of 99 predominantly Northern European patients with NAFLD (age range of 20-77 years) were studied to perform this secondary analysis of baseline characteristics of patients recruited to the INSYTE (Investigation of Synbiotic Treatment in NAFLD) trial (www.clinicaltrials.gov registered number NCT01680640). These patients were recruited as described in detail previously $[20,21]$. The trial design was approved by the Southampton and South West Hampshire research ethics committee (12/ SC/0614). All patients gave their written informed consent.

\section{Inclusion and exclusion criteria}

The inclusion and exclusion criteria for the INSYTE trial have been previously described in detail [20,21]. Briefly, participants were aged $>18$ years with a diagnosis of NAFLD confirmed in secondary care, with evidence of hepatic steatosis confirmed by via proton magnetic resonance spectroscopy ( $\left.{ }^{1} \mathrm{H}-\mathrm{MRS}\right)$ at recruitment.

\section{Anthropometric and biochemical measurements}

Anthropometric and biochemical measurements were collected as previously described [20,21]. Body composition was assessed by dual-energy X-ray absorptiometry (DEXA). Blood pressure was measured using a Marquette Dash 300 monitor (GE Healthcare, Little Chalfont, Bucks, UK) as previously described [21]. Handgrip strength was measured using a Jamar hand Dynamometer with participants seated and their arms rested on the chair arms - data are presented as grip strength (kg). Fasting glucose, haemoglobin A1c (HbA1c), fasting insulin, total cholesterol, high-density lipoprotein (HDL)-cholesterol, triglycerides, alanine aminotransferase (ALT), aspartate aminotransferase (AST), adiponectin, leptin, high-sensitivity C-reactive protein (hs-CRP), tumour necrosis factor-a (TNFa), interleukin (IL)-6, IL-8 and IL-10 concentrations were measured in serum samples using commercially available kits according to the manufacturer's instructions. Serum lipopolysaccharide (LPS) concentrations were measured as previously described [20]. Concentrations of GDF-15, leptin and adiponectin were measured in fasting serum samples by the Cambridge Biochemical Assay Laboratory, University of Cambridge. Serum GDF-15 quantification was done with antibodies and standards from R\&D Systems (R\&D Systems - catalogue number DY957) and in accordance with manufacturer's instructions and as previously described [18]. The estimated glomerular filtration rate (e-GFR) was measured using the CKD-Epidemiology Collaboration (CKD-EPI) study equation [22]. Satiety hormones, such as plasma ghrelin, gastric inhibitory polypeptide (GIP), glucagon-like peptide (GLP-1), peptide YY (PYY) and pancreatic peptide (PP) concentrations were also measured as previously described [21] using the MILLIPLEX ${ }^{\circledR}$ MAP Human Metabolic Hormone Panels Kit.

\section{Liver fat and vibration-controlled transient elastography measurements}

Liver fat and VCTE-derived kPa measurements were collected as previously described $[20,21]$. In all participants, the quantification of intra-hepatic fat content was undertaken via proton magnetic resonance spectroscopy $\left({ }^{1} \mathrm{H}\right.$ MRS) (see Supplementary Material for method). Liver VCTE-derived $\mathrm{kPa}$ measurements were assessed as a clinically recognised proxy measure of liver elasticity using the Echosens (Waltham, MA) Fibroscan ${ }^{\circledR}$ by a trained clinician (ES). Results are expressed as the medians (IQRs) in kilo-pascals $(\mathrm{kPa})$. Liver VCTE-derived $\mathrm{kPa}$ measurements of $\geq 8.2 \mathrm{kPa}$ and $\geq 9.7 \mathrm{kPa}$ were used as validated proxy thresholds for identification $\geq \mathrm{F} 2$ and $\geq \mathrm{F} 3$ fibrosis with the former having a AUROC of $0.77(95 \% \mathrm{Cl} 0.72,0.82)$ for the prediction of $\geq \mathrm{F} 2$ fibrosis (sensitivity and specificity $=71 \%$ and $70 \%$, respectively) as recently reported [23]. The technical description and examination procedures for liver VCTE-kPa measurements have also been previously described [24].

\section{Appetite, hunger and satiety assessment}

Assessment of patient appetite, hunger and satiety was done as previously described $[20,21]$. See Supplementary Material for further description of this methodology.

\section{Gut microbiota analyses-DNA extraction, sequencing and bioinformatics}

Gut microbiota DNA extraction from faecal samples, 165 amplicon sequencing and bioinformatic analyses were performed as previously described [20, 21]. See Supplementary Material for further details of this methodology.

\section{Statistical analysis}

Data were analysed using Statistical Package for the Social Sciences (SPSS) Version 26.0 (New York, USA). Data were tested for normality using the Shapiro-Wilk and Kolmogorov-Smirnov tests and are presented as means (SD) for normally distributed variables and medians and inter-quartile ranges (IQRs) for non-normally distributed variables. Comparisons of continuous variables between groups were performed with the unpaired Student $t$-test for normally distributed variables and the Mann-Whitney $U$ test for non-normally distributed variables, and differences in proportions were investigated using the chi-squared test or the Fisher's exact test as appropriate. Univariable associations between variables were investigated using Pearson's correlation for normally distributed or Spearman's rank correlation for non-normally distributed variables. To test for the independence of associations between explanatory factors and serum GDF-15 concentrations, VCTE-measured liver $\mathrm{kPa}$ measurements or additional liver fibrosis biomarkers, factors were entered into a multivariable linear regression model with either: (a) logarithmically transformed GDF-15 concentrations; (b) logarithmically transformed liver kPa measurements; (c) Enhanced liver fibrosis (ELF) scores; (d) logarithmically transformed Fibrosis-4 (FIB-4) scores; (e) logarithmically transformed AST to platelet ratio index (APRI) scores or (f) hepatic mitochondrial function (HMF) (as determined by the 13C-ketoisocaproate breath test [13C-KICA BT]) as the outcome variable. Regression models were run with all explanatory factors, or stepwise, to investigate the proportion of total variance in serum GDF-15 concentrations that could be explained by each individual explanatory factor. Binary logistic regression modelling was used to investigate whether serum GDF-15 concentrations and/or T2DM status were independently associated with $\geq F 2$ and $\geq F 3$ fibrosis (as indicated by the validated VCTE measurement of $\geq 8.2$ and $\geq 9.7 \mathrm{kPa}$, respectively), to identify whether other liver fibrosis biomarkers were associated with $\geq F 2$ and $\geq \mathrm{F} 3$ fibrosis and also to identify factors that were independently associated with high $(\geq 1193.7 \mathrm{pg} / \mathrm{ml})$ serum GDF-15 concentrations. Goodness of fit for the models was tested with Hosmer-Lemeshow tests. Sobel test statistics and $p$-values were calculated to test the potential involvement of GDF-15 concentrations in the association between T2DM and either $\geq F 2$, or $\geq F 3$ fibrosis separately according to [25]. Receiveroperating characteristic (ROC) curve analysis for GDF-15 or HbA1c was performed to estimate areas under the receiver-operating characteristic curves (AUROCs), as well as to estimate the best cut-off values (Youden's index) to predict $\geq F 2$ and $\geq F 3$ fibrosis, or high serum GDF-15 concentrations. See Supplementary Material for methods used for the statistical analysis of the gut microbiota. Where data were not available on all 99 participants, the number of subjects included in the analysis for which there was complete data are presented in the relevant table or figure legend.

\section{RESULTS}

\section{Characteristics of participants}

The mean (SD) age of the 99 patients with NAFLD (61 men, 38 women) included in the study was 50.9 (12.8) years. Table 1 shows the characteristics of patients, stratified by T2DM status. In patients with NAFLD and T2DM, liver VCTE-derived kPa measurements were significantly higher and there was a greater prevalence of $\geq F 2$ and $\geq F 3$ liver fibrosis, (according to the previously validated VCTE thresholds of $\geq 8.2 \mathrm{kPa}$ and $\geq 9.7 \mathrm{kPa}$, respectively) compared to counterparts without T2DM. Furthermore, FIB-4 scores were significantly higher in patients with both NAFLD and T2DM. Fasting glucose and $\mathrm{HbA1C}$ concentrations were also higher in patients with NAFLD and T2DM, whereas 
Table 1. Characteristics of patients with NAFLD stratified by preexisting type 2 diabetes status.

\begin{tabular}{|c|c|c|c|}
\hline Variables & $\begin{array}{l}\text { Without } \\
\text { T2DM }(n=57)\end{array}$ & $\begin{array}{l}\text { With T2DM } \\
(n=42)\end{array}$ & $p$-value \\
\hline Age (yrs) & $48.7 \pm 14.2$ & $53.8 \pm 10.1$ & 0.04 \\
\hline Sex (male) $(n, \%)^{\mathrm{a}}$ & $38(66.7 \%)$ & $23(54.8 \%)$ & 0.23 \\
\hline $\begin{array}{l}\text { Smoking history (no) } \\
(n, \%)^{\mathrm{a}}\end{array}$ & $53(93 \%)$ & $34(81 \%)$ & 0.12 \\
\hline $\begin{array}{l}\text { Systolic blood } \\
\text { pressure }(\mathrm{mmHg})\end{array}$ & $133.7(19.5)$ & $134.4(27.5)$ & 0.8 \\
\hline $\begin{array}{l}\text { Diastolic blood } \\
\text { pressure }(\mathrm{mmHg})\end{array}$ & $74.4 \pm 8.3$ & $73.9 \pm 10.7$ & 0.82 \\
\hline BMI $\left(\mathrm{kg} / \mathrm{m}^{2}\right)$ & $32.3(6.4)$ & $34.9(6.8)$ & 0.06 \\
\hline $\begin{array}{l}\text { DEXA lean body } \\
\text { mass }(\mathrm{kg})\end{array}$ & $64.5 \pm 13.2$ & $62 \pm 10.6$ & 0.25 \\
\hline $\begin{array}{l}\text { DEXA total body } \\
\text { fat }(\%)\end{array}$ & $33.8(10.2)$ & $36.7(12.2)$ & 0.22 \\
\hline $\begin{array}{l}\text { Handgrip } \\
\text { strength (kg) }\end{array}$ & $36.7(25.8)$ & $31.2(19.5)$ & 0.05 \\
\hline $\begin{array}{l}\text { Fasting glucose } \\
(\mathrm{mmol} / \mathrm{l})\end{array}$ & $5.3(1.0)$ & $8.1(3.3)$ & $<0.0001$ \\
\hline $\begin{array}{l}\text { Haemoglobin A1c } \\
(\mathrm{mmol} / \mathrm{mol})\end{array}$ & $35.0(5.5)$ & $59.5(23)$ & $<0.0001$ \\
\hline $\begin{array}{l}\text { Fasting insulin (mIU/ } \\
\mathrm{L}^{\mathrm{b}}\end{array}$ & $14.2(9.7)$ & $13.7(9.0)$ & 0.94 \\
\hline HOMA-IR ${ }^{c}$ & $3.5(2.7)$ & $5.4(5.1)$ & $<0.001$ \\
\hline $\begin{array}{l}\text { Metformin use (yes) } \\
(n, \%)^{\mathrm{a}}\end{array}$ & $0(0 \%)$ & $29(69 \%)$ & $<0.0001$ \\
\hline Triglycerides (mmol/l) & $1.8(1)$ & $1.8(1.2)$ & 0.27 \\
\hline $\begin{array}{l}\text { Total cholesterol } \\
(\mathrm{mmol} / \mathrm{l})\end{array}$ & $5.2(1.4)$ & $4.4(1.3)$ & 0.001 \\
\hline $\begin{array}{l}\text { HDL cholesterol } \\
(\mathrm{mmol} / \mathrm{l})\end{array}$ & $1.2(0.4)$ & $1.2(0.3)$ & 0.61 \\
\hline AST (IU/I) & $34.0(22.0)$ & $38.0(31.5)$ & 0.54 \\
\hline ALT (IU/I) & $56.0(45.0)$ & $59.0(41.8)$ & 0.8 \\
\hline $\begin{array}{l}\text { MRS-measured liver } \\
\text { fat (\%) }\end{array}$ & $23.7(34.8)$ & $27.0(24.1)$ & 0.871 \\
\hline $\begin{array}{l}\text { 13C-KICA BT (CPDR } \\
\text { over } 1 \mathrm{~h}-\%)\end{array}$ & $14.5 \pm 3.7$ & $12.6 \pm 3.2$ & 0.008 \\
\hline Liver VCTE $(\mathrm{kPa})^{\mathrm{c}}$ & $6.0(3.1)$ & $8.0(4.8)$ & 0.01 \\
\hline $\begin{array}{l}\text { Liver VCTE } \geq 8.2 \mathrm{kPa} \text {, } \\
\text { (yes) }(\%)^{\mathrm{a}}\end{array}$ & $12(23.2 \%)$ & $20(48.8 \%)$ & 0.01 \\
\hline $\begin{array}{l}\text { Liver VCTE } \geq 9.7 \mathrm{kPa} \text {, } \\
\text { (yes) }(\%)^{\mathrm{a}}\end{array}$ & 7 (13.5\%) & 15 (36.6\%) & 0.009 \\
\hline $\mathrm{APRI}^{\mathrm{c}}$ & $0.4(0.3)$ & $0.4(0.5)$ & 0.44 \\
\hline FIB- $4^{c}$ & $0.9(1.2)$ & $1.2(1.1)$ & 0.02 \\
\hline $\mathrm{ELF}^{\mathrm{d}}$ & $6.9 \pm 0.4$ & $7.0 \pm 0.3$ & 0.06 \\
\hline GDF-15 (pg/ml) & $640.3(332.5)$ & $1271.0(902.1)$ & $<0.0001$ \\
\hline Adiponectin $(\mu \mathrm{g} / \mathrm{ml})$ & $4.9(2.4)$ & $3.8(2.6)$ & 0.31 \\
\hline Leptin $(\mathrm{ng} / \mathrm{ml})^{\mathrm{d}}$ & $20.0(32.9)$ & $24.4(31.2)$ & 0.77 \\
\hline $\mathrm{TNF} \alpha(\mathrm{pg} / \mathrm{ml})^{\mathrm{d}}$ & $12.9(5.5)$ & $10.4(4.1)$ & 0.15 \\
\hline IL-6 (pg/ml) & $2.6(1.6)$ & $2.6(2.0)$ & 0.26 \\
\hline IL-8 (pg/ml) & $13.8(7.2)$ & $17.8(10.5)$ & 0.01 \\
\hline IL-10 (pg/ml) & $0.8(0.4)$ & $0.7(0.4)$ & 0.89 \\
\hline hs-CRP (mg/l) & $2.0(3.0)$ & $4.0(5.3)$ & 0.003 \\
\hline LPS (EU/ml) & $0.2(0.1)$ & $0.1(0.1)$ & 0.51 \\
\hline $\begin{array}{l}\text { e-GFR }(\mathrm{ml} / \mathrm{min} / 1.73 \\
\left.\mathrm{m}^{2}\right)\end{array}$ & $90.0(12.3)$ & $90.0(9.8)$ & 0.68 \\
\hline
\end{tabular}

Data presented as means \pm SDs or medians (inter-quartile ranges).

T2DM Type 2 diabetes mellitus, BMI body mass index, DEXA dual-energy $\mathrm{X}$ ray absorptiometry, HOMA-IR homeostatic model assessment of insulin resistance, $H D L$ high-density lipoprotein, AST aspartate aminotransferase, ALT alanine transaminase, MRS magnetic resonance spectroscopy, 13C-KICA $B T$ 13C-ketoisocaproate breath test, VCTE vibration-controlled transient elastography, APRI AST to platelet ratio index, FIB-4 Fibrosis-4, ELF enhanced liver fibrosis GDF-15 growth differentiation factor-15, TNFa tumour necrosis factor- $\alpha$, IL interleukin, $h s$-CRP high-sensitivity C-reactive protein, LPS lipopolysaccharide, e-GFR estimated glomerular filtration rate.

${ }^{a}$ Cross-tab. Pearson chi-squared test.

${ }^{\mathrm{b}}$ Data were only available for no T2DM $n=52$ and T2DM $n=32$.

${ }^{\mathrm{C}}$ Data were only available for no T2DM $n=52$ and T2DM $n=41$.

${ }^{\mathrm{d} D a t a}$ were only available for no T2DM $n=50$ and T2DM $n=38$.

fasting insulin concentrations were not different between groups. Twenty-nine $(69 \%)$ patients with T2DM were receiving metformin treatment. In patients with NAFLD and T2DM, age, HOMA-IR, IL-8 and hs-CRP were also higher than in those without T2DM whereas HMF was lower in patients with both NAFLD and T2DM. Notably, serum GDF-15 concentrations were markedly higher in patients with NAFLD and T2DM than in those without T2DM (Table 1 and Fig. 1a). Regarding gut microbiota composition, the relative abundance of the Enterobacteriaceae family was greater in patients with NAFLD and T2DM than in those without T2DM ( $p$ $=0.001$, data not shown). In patients with NAFLD and T2DM, fasting ghrelin concentrations were lower and fasting GLP-1 concentrations were higher, compared to patients without T2DM (Supplementary Table 1). Furthermore, following a breakfast challenge, ghrelin AUC was lower and GLP-1, PP and PYY AUCs were higher in patients with NAFLD and T2DM, than in their counterparts without T2DM (Supplementary Table 1). However, neither fasted nor AUC values, for reported hunger, fullness, and satiety, were significantly different in patients with or without T2DM (Supplementary Table 1).

\section{Serum GDF-15 concentrations predict liver fibrosis and are potentially involved in the association between T2DM and liver fibrosis}

In univariable analysis, higher serum GDF-15 concentrations were associated with higher liver VCTE-derived kPa measurements (Fig. $1 \mathrm{~b})$, and serum GDF-15 concentrations were higher in patients with $\geq$ F2 fibrosis (Fig. 1c). Both APRI and FIB-4 test scores were positively associated with the presence of $\geq \mathrm{F} 2$ and $\geq \mathrm{F} 3$ fibrosis independently of age and sex (Supplementary Table 2). Similarly, serum GDF-15 was significantly and positively associated with APRI, FIB-4 and ELF test scores (Table 2). Serum GDF-15 concentrations were associated with higher concentrations of fasting glucose (Table 2) and HbA1c (Fig. 1d) $(r=0.60$, and $r=$ 0.62 , respectively, $p<0.00001$ for both). Serum GDF-15 concentrations were also positively associated with age, BMI, total body fat percentage, HOMA-IR, AST, IL-6, IL-8, hs-CRP concentrations, and negatively with e-GFR, HMF and handgrip strength (Table 2).

Multivariable linear regression modelling was undertaken to investigate which factors were independently associated with liver VCTE-derived $\mathrm{kPa}$ measurements as the outcome variable. In a regression model where GDF-15, age, sex, total body fat, T2DM status, e-GFR and AST were entered as putative key explanatory variables and liver $\mathrm{kPa}$ measurement as the outcome, only serum GDF-15 concentrations were independently associated with liver $\mathrm{kPa}$ measurement [unstandardised $\beta$ coefficient $=0.35(95 \% \mathrm{Cl}$ $0.15-0.56$ ), $p=0.001$ (model fit $R^{2}=0.261 ; p=0.001$ )] (Supplementary Table 3). In this regression model, GDF-15 concentrations alone explained $21 \%$ of the total variance in liver VCTE-derived $\mathrm{kPa}$ value. Intriguingly, upon removal of GDF-15 from this model, T2DM status became independently associated with liver VCTEderived $\mathrm{kPa}$ measurement $[\beta$ coefficient $=0.09(0.18-0.17), p=$ 0.015], whereas age, sex, total body fat, e-GFR and AST were not 

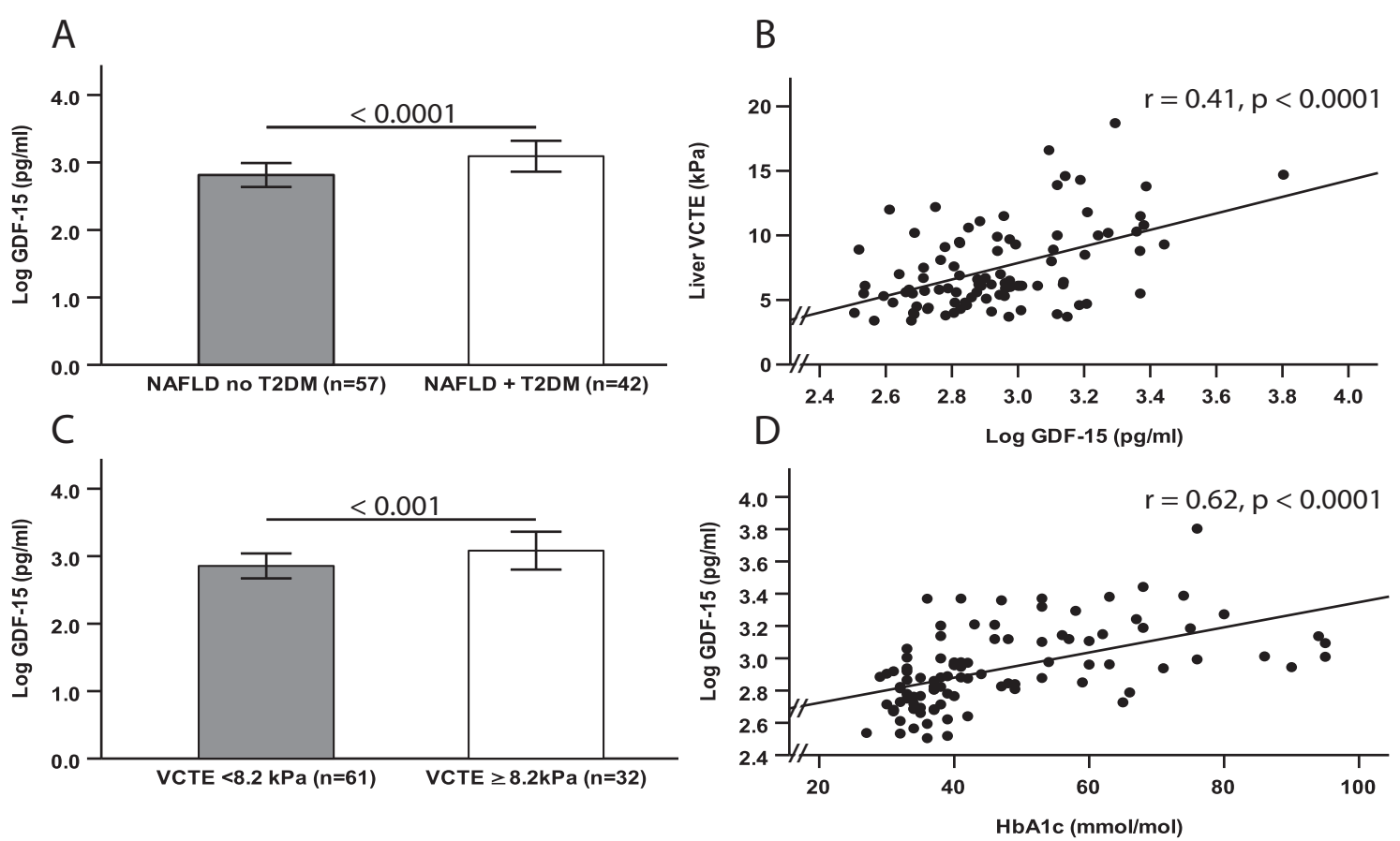

Fig. 1 Differences in serum GDF-15 concentrations by type 2 diabetes status, predicted liver fibrosis severity and scatter plots showing the association between serum GDF-15 concentrations and both haemoglobin A1c and liver stiffness measurements (as assessed by vibration-controlled transient elastography [VCTE]). A The differences in serum GDF-15 concentrations (logarithmically transformed) between NAFLD patients with and without coexisting type 2 diabetes. B The scatter plot for the association of serum GDF-15 concentrations with liver VCTE measurements $(\mathrm{kPa})$. C The differences in serum GDF-15 concentrations between NAFLD patients with $<\mathrm{F} 2$ or $\geq \mathrm{F} 2$ fibrosis according to the validated VCTE measurement threshold of $\geq 8.2 \mathrm{kPa}$ as a proxy for the identification of $\geq F 2$ fibrosis. D The scatter plot for the association of serum GDF-15 with HbA1c concentrations. Data are presented as mean \pm SD. Associations are Spearman's rank correlation coefficients. Sample size A, D $n=99 ; \mathbf{B}, \mathbf{C} n=93$. VCTE vibration-controlled transient elastography, GDF-15 growth differentiation factor-15, T2DM type 2 diabetes mellitus.

associated with liver VCTE-derived kPa measurement (model fit $R^{2}$ $=0.152 ; p=0.03$ ). Serum GDF-15 concentrations were also positively and independently associated with ELF, FIB-4 and APRI scores and, according to stepwise analysis, contributed the most towards the total variance of each liver fibrosis biomarker (Supplementary Table 3). Serum GDF-15 concentrations were not independently associated with HMF in a model with the same combination of explanatory variables (data not shown).

Since we found that serum GDF-15 concentrations were independently associated with liver VCTE-derived $\mathrm{kPa}$ measurements, and alone explained $21 \%$ of the variance in liver VCTEderived $\mathrm{kPa}$ measurements, we next tested whether GDF-15 concentrations and/or T2DM status could predict $\geq \mathrm{F} 2$ fibrosis, as determined by the validated VCTE threshold of $\geq 8.2 \mathrm{kPa}$ [23]. In a model that did not include serum GDF-15 concentrations, T2DM status was associated with $\geq F 2$ fibrosis (Table 3 - model 1). However, when both T2DM status and serum GDF-15 concentrations were added as covariates and $\geq F 2$ fibrosis status was the outcome, only GDF-15 concentration was associated with $\geq F 2$ fibrosis (Table 3 - model 2). Furthermore, in a fully adjusted model where T2DM, GDF-15 concentrations, age, sex, total body fat percentage, e-GFR and AST concentrations were entered as key covariates (identified from multivariable linear regression modelling see Supplementary Table 3 ) and $\geq F 2$ fibrosis status was the outcome variable, only serum GDF-15 concentrations were associated with $\geq F 2$ fibrosis (Table 3 - model 3). Goodness of fit for the models was tested with Hosmer-Lemeshow tests. A model that only included GDF-15 concentrations as the explanatory variable showed excellent goodness of fit (chi-squared statistic $=$ $2.71, p=0.95$ ). Additionally, as HOMA-IR was significantly higher in NAFLD patients with T2DM compared to those without T2DM and insulin resistance may be an important factor in the relationship between T2DM and liver fibrosis, we explored whether GDF-15 concentrations were associated with liver fibrosis severity, independently of HOMA-IR. In a model where $\geq F 2$ fibrosis status was the outcome and HOMA-IR, T2DM status and GDF-15 concentrations were the explanatory variables, only GDF-15 concentration (and not HOMA-IR or T2DM) was associated with $\geq F 2$ fibrosis $[\mathrm{OR}=1.002(1.001-1.003), p=0.004$, for each $1 \mathrm{pg} / \mathrm{ml}$ of GDF-15]. Furthermore, we found strikingly similar results for $\geq F 3$ fibrosis status (data not shown but available from the authors).

Given this result, we performed ROC curve analysis to assess the ability of GDF-15 concentrations to predict the presence of $\geq F 2$ fibrosis and to identify an optimal GDF-15 concentration cut-off for predicting $\geq F 2$ fibrosis. Accordingly, the AUROC for the prediction of $\geq \mathrm{F} 2$ fibrosis was 0.75 (95\% Cl $0.63-0.86, p<0.001)$. The Youden index (optimal cut-off) GDF-15 concentration was $1193.7 \mathrm{pg} / \mathrm{ml}$ with sensitivity, specificity, positive predictive value (PPV) and negative predictive value (NPV) of $56.3 \%, 86.9 \%, 69.2 \%$ and $79.1 \%$, respectively (Fig. 2 ). We then repeated the ROC curve analysis using the higher validated liver $\mathrm{kPa}$ threshold for $\geq \mathrm{F} 3$ fibrosis $(\geq 9.7 \mathrm{kPa})$, as the binary outcome. As shown in Supplementary figure 1, these results were remarkably similar to those obtained for $\geq F 2$ fibrosis [AUROC 0.762 (95\% Cl 0.64-0.89), $p<0.0001$, optimal cut-off of serum GDF-15 $1193.7 \mathrm{pg} / \mathrm{ml}$; sensitivity $63.6 \%$, specificity $83.1 \%$, PPV $53.8 \%$ and NPV $88.1 \%]$. In order to assess whether circulating concentrations of GDF-15 were potentially involved in the relationship between T2DM and either $\geq F 2$ fibrosis or $\geq F 3$ fibrosis, we next calculated Sobel test statistics and $p$-values. These data suggested that GDF-15 was potentially involved in the associations between T2DM and $\geq F 2$ fibrosis as well as between T2DM and $\geq F 3$ fibrosis (Sobel test statistics 2.90, $p=0.004$; and 2.71, $p=0.007$, for $\geq \mathrm{F} 2$ fibrosis and $\geq \mathrm{F} 3$ fibrosis, respectively). 
Table 2. Univariable linear associations with serum GDF-15 concentrations.

\begin{tabular}{|c|c|c|}
\hline Variables & $\begin{array}{l}\text { Correlation coefficient } \\
\text { (s) }\end{array}$ & $p$-value \\
\hline Age (yrs) & 0.44 & $<0.00001$ \\
\hline $\begin{array}{l}\text { Systolic blood } \\
\text { pressure }(\mathrm{mmHg})\end{array}$ & 0.05 & 0.65 \\
\hline $\begin{array}{l}\text { Diastolic blood } \\
\text { pressure }(\mathrm{mmHg})\end{array}$ & 0.06 & 0.56 \\
\hline BMI $\left(\mathrm{kg} / \mathrm{m}^{2}\right)^{\mathrm{a}}$ & 0.29 & 0.003 \\
\hline DEXA lean body mass $(\mathrm{kg})$ & -0.15 & 0.16 \\
\hline DEXA total body fat (\%) & 0.22 & 0.03 \\
\hline Handgrip strength $(\mathrm{kg})$ & -0.31 & 0.002 \\
\hline Fasting glucose $(\mathrm{mmol} /)^{a}$ & 0.60 & $<0.00001$ \\
\hline $\begin{array}{l}\text { Haemoglobin A1c (mmol/ } \\
\text { mola })^{\mathrm{a}}\end{array}$ & 0.62 & $<0.00001$ \\
\hline Fasting insulin $(\mathrm{mlU} / \mathrm{L})^{\mathrm{b}}$ & 0.11 & 0.32 \\
\hline HOMA-IR & 0.41 & $<0.0001$ \\
\hline Triglycerides $(\mathrm{mmol} / \mathrm{l})^{a}$ & 0.20 & 0.84 \\
\hline Total cholesterol $\left(\mathrm{mmol} / \mathrm{l}^{\mathrm{a}}\right.$ & -0.29 & 0.003 \\
\hline $\mathrm{HDL}$ cholesterol $\left(\mathrm{mmol} / \mathrm{l}^{\mathrm{a}}\right.$ & -0.01 & 0.94 \\
\hline AST $(I U / I)^{a}$ & 0.29 & 0.003 \\
\hline $\operatorname{ALT}(I U / I)^{a}$ & 0.13 & 0.19 \\
\hline MRS-measured liver fat (\%) ${ }^{\mathrm{a}}$ & -0.10 & 0.35 \\
\hline $\begin{array}{l}\text { 13C-KICA BT (CPDR over 1h- } \\
\%)^{\mathrm{a}}\end{array}$ & -0.38 & $<0.001$ \\
\hline Liver VCTE $(\mathrm{kPa})^{\mathrm{a}, \mathrm{c}}$ & 0.41 & $<0.0001$ \\
\hline$A P R I^{a, c}$ & 0.28 & 0.007 \\
\hline $\mathrm{FIB}-4^{\mathrm{a}, \mathrm{c}}$ & 0.53 & $<0.00001$ \\
\hline $\mathrm{ELF}^{\mathrm{a}, \mathrm{d}}$ & 0.53 & $<0.00001$ \\
\hline Adiponectin $(\mu \mathrm{g} / \mathrm{ml})^{d}$ & -0.02 & 0.85 \\
\hline Leptin $(\mathrm{ng} / \mathrm{ml})^{\mathrm{d}}$ & 0.16 & 0.14 \\
\hline $\mathrm{TNF} \alpha(\mathrm{pg} / \mathrm{ml})^{\mathrm{a}}$ & 0.06 & 0.57 \\
\hline IL-6 (pg/ml) $)^{a}$ & 0.25 & 0.02 \\
\hline IL-8 (pg/ml) ${ }^{\mathrm{a}}$ & 0.33 & 0.001 \\
\hline $\mathrm{IL}-10(\mathrm{pg} / \mathrm{ml})^{\mathrm{a}}$ & 0.19 & 0.07 \\
\hline hs-CRP (mg/l) ${ }^{a}$ & 0.32 & 0.001 \\
\hline LPS (EU/ml) & 0.12 & 0.25 \\
\hline e-GFR $\left(\mathrm{ml} / \mathrm{min} / 1.73 \mathrm{~m}^{2}\right)^{\mathrm{a}}$ & -0.24 & 0.018 \\
\hline \multicolumn{3}{|c|}{$\begin{array}{l}\text { Sample size, } n=99 \text {. } \\
B M I \text { body mass index, DEXA dual-energy X-ray absorptiometry, } H D L \text { high- } \\
\text { density lipoprotein, AST aspartate aminotransferase, ALT alanine transami- } \\
\text { nase, MRS magnetic resonance spectroscopy, 13C-KICA BT 13C- } \\
\text { ketoisocaproate breath test, VCTE vibration-controlled transient elastogra- } \\
\text { phy, APRI AST to platelet ratio index, FIB-4 Fibrosis-4, ELF enhanced liver } \\
\text { fibrosis, GDF-15 growth differentiation factor-15, TNFa tumour necrosis } \\
\text { factor- } \alpha, L \text { interleukin, hs-CRP high-sensitivity C-reactive protein, e-GFR } \\
\text { estimated glomerular filtration rate. } \\
\text { aspearman's rank correlation coefficients. } \\
\text { bSample size } 82 \text {. } \\
\text { 'Samples size } n=93 \text {. } \\
\text { dSample size } 88 .\end{array}$} \\
\hline
\end{tabular}

HbA1c levels are independently associated with, and can predict high GDF-15 concentrations in patients with NAFLD Given that very little is known regarding the potential regulatory factors of elevated GDF-15 in patients with NAFLD and T2DM, we next looked to identify the factors associated with T2DM that were independently associated with GDF-15 concentrations. GDF-15
Table 3. Binary logistic regression analysis showing that only serum GDF-15 concentrations and T2DM status were significant independent predictors of a predicted liver fibrosis severity of $\geq F 2$ (as measured by VCTE).

\begin{tabular}{|c|c|c|}
\hline Variables & OR $(95 \% \mathrm{Cl})$ & $P$-value \\
\hline \multicolumn{3}{|l|}{ Model 1} \\
\hline T2DM status & $3.18(1.3-7.72)$ & 0.01 \\
\hline \multicolumn{3}{|l|}{ Model 2} \\
\hline T2DM status & $1.16(0.39-3.48)$ & 0.79 \\
\hline Serum GDF-15 (pg/ml) & $1.002(1.001-1.003)$ & 0.001 \\
\hline \multicolumn{3}{|l|}{ Model $3^{a}$} \\
\hline T2DM status & $1.30(0.4-4.2)$ & 0.72 \\
\hline Serum GDF-15 (pg/ml) & 1.002 (1.001-1.003) & 0.006 \\
\hline
\end{tabular}

Model 1 only contains T2DM status. Note: GDF-15 ORs are for each $1 \mathrm{pg} / \mathrm{ml}$ of GDF-15. Dependent variable was liver VCTE measurements $<8.2 \mathrm{kPa}$ vs. $\geq 8.2 \mathrm{kPa}$ ( 0 and 1 , respectively) as a proxy threshold for the identification of $\geq$ F2 fibrosis. Sample size $n=93$.

T2DM type 2 diabetes mellitus, AST aspartate aminotransferase, VCTE vibration-controlled transient elastography, GDF-15 growth differentiation factor-15, e-GFR estimated glomerular filtration.

${ }^{a}$ Model is adjusted for age, sex, total body fat percentage, e-GFR, and AST concentrations.

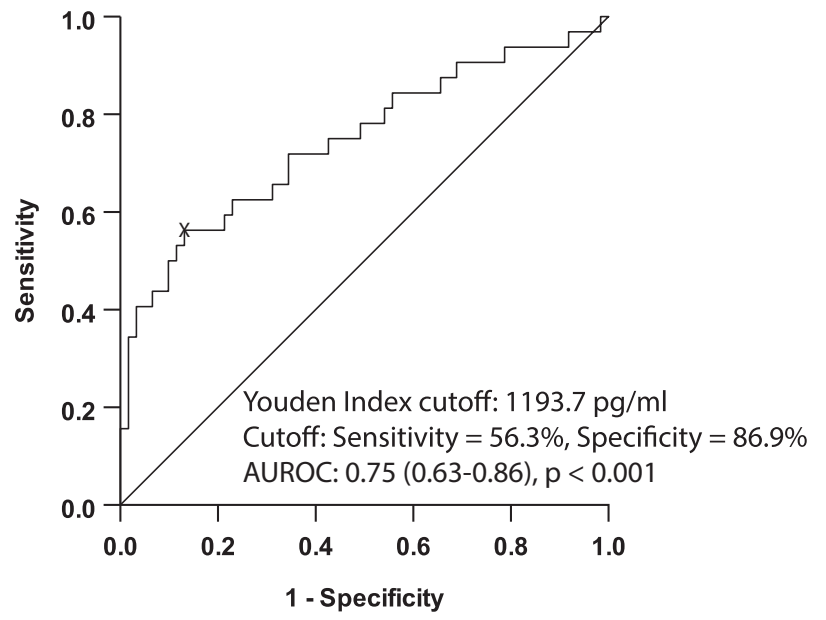

Fig. 2 Receiver-operating characteristic curve of serum GDF-15 concentrations for $\geq F 2$ fibrosis ( $\geq 8.2 \mathrm{kPa}$ as measured by VCTE). Sample size $n=93$.

concentrations were higher in patients treated with metformin, compared to those not receiving metformin $(p<0.0001)$ (Fig. 3). In univariable analyses, we did not find any significant associations between participant-reported measures of satiety and/or plasma concentrations of satiety hormones with GDF-15 concentrations (Supplementary Table 4). Similarly, none of the measures of satiety (AUC values) was associated with GDF-15 concentrations except for GLP-1 AUC, which was positively associated with GDF-15 concentrations ( $r=0.30, p=0.003)$. However, this association was no longer significant after controlling for metformin use $(r=0.06$, $p=0.57)$. Within the faecal microbiota, there was a greater relative abundance of the Enterobacteriaceae family in the high vs. the low GDF-15 concentration tertile $(q=0.003)$. Similarly, according to univariable correlation analyses, serum GDF-15 concentrations were associated with the abundance of Enterobacteriaceae family $(r=0.52, p<0.0001)$ in faecal samples. However, in multivariable regression modelling, this family of bacteria was not independently associated with GDF-15 concentrations (data not shown). 
In regression modelling, the explanatory factors that were independently (all $p=0.01$ or less) associated with higher GDF-15 concentrations were as follows: higher $\mathrm{HbA} 1 \mathrm{c}$, older age, higher AST, metformin treatment, higher hs-CRP and lower e-GFR (model fit $R^{2}=0.60, p<0.00001$ ) (Table 4). Collectively, these factors together explained $60 \%$ of the total variance in GDF-15 concentrations. None of the measures of appetite, hunger and/ or satiety was independently associated with GDF-15 concentrations (data not shown). As we were able to explain a substantial proportion of the total variance in GDF-15 concentrations within the present cohort, we undertook stepwise linear regression modelling to investigate the proportion of the variance in GDF-15 concentrations that could be explained by each of the aforementioned independent factors. In doing so, we found $\mathrm{HbA} 1 \mathrm{c}$ alone explained $29.6 \%$ of the total variance in GDF-15 concentrations (Table 4, model 1). The addition of age (model 2 ) led to a statistically significant increase in $R^{2}$ of $0.102(p<0.001)$, AST and increase in $R^{2}$ of $0.083(p<0.001)$ (model 3), metformin use an increase in $R^{2}$ of $0.062(p=0.001)$ (model 4$)$, hs-CRP an increase in $R^{2}$ of $0.03(p=0.013)$ (model 5$)$ and e-GFR an increase in $R^{2}$ of $0.028(p=0.015)$ (model 6$)$. Thus, the addition of each of these independent factors explained a further $10.2 \%$ (age), $8.3 \%$ (AST), $6.2 \%$ (metformin treatment), 3.0\% (hs-CRP) and 2.8\% (e-GFR), respectively, compared to the $29.6 \%$ of the total variance in GDF15 concentrations, explained by $\mathrm{HbA} 1 \mathrm{c}$ alone.

We next tested whether increased $\mathrm{HbA} 1 \mathrm{c}$ concentration was associated with a high GDF-15 concentration using a GDF-15

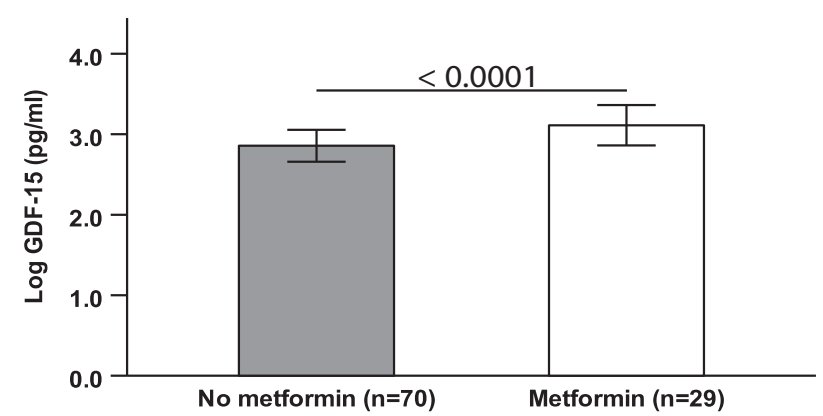

Fig. 3 Differences in serum GDF-15 concentrations in patients with NAFLD not receiving vs. receiving metformin treatment. Data are presented as means \pm SD. threshold of $\geq 1193.7 \mathrm{pg} / \mathrm{ml}$ that we had identified as the optimal cut-off for the prediction of $\geq F 2$ fibrosis (Fig. 2). We carried out binary logistic regression modelling where, in the first model, only $\mathrm{HbA1C}$ concentration was entered as a covariate and GDF-15 concentrations were entered as the binary outcome $[<1193.7 \mathrm{pg} /$ $\mathrm{ml}(n=72)$ vs. $\geq 1193.7 \mathrm{pg} / \mathrm{ml}(n=27)]$. In this regression model, higher HbA1c was associated GDF-15 concentrations (OR, 1.07; 95\% Cl 1.0-2.0; $p<0.0001$ ) (Supplementary Table 5 - model 1). In the final adjusted model where $\mathrm{HbA} 1 \mathrm{c}$, age, metformin treatment, hs-CRP, AST and e-GFR were entered as covariates, and GDF-15 concentrations were entered as the binary outcome, higher $\mathrm{HbA1c}$, metformin use, higher hs-CRP, higher AST and lower e-GFR were all independently associated with higher GDF-15 concentrations (model 2). Next, we carried out a ROC curve analysis to assess whether HbA1c predicted high GDF-15 concentrations. The AUROC for the prediction of GDF-15 concentrations was 0.83 (95\% $\mathrm{Cl} 0.75-0.91)$ and the optimal cut-off $\mathrm{HbA} 1 \mathrm{c}$ concentration was $42.5 \mathrm{mmol} / \mathrm{mol}$ (sensitivity, specificity, PPV and NPV were $85.2 \%, 76.4 \%, 57.5 \%$ and $93.2 \%$, respectively) for the prediction of GDF-15 concentrations (Fig. 4).

\section{DISCUSSION}

The novel findings in this study are that in patients with NAFLD, $\mathrm{HbA1C}$ concentrations explain a large proportion ( 30\%) of the variance in GDF-15 concentrations and that circulating concentration of GDF-15 may be involved in the known association between T2DM and liver fibrosis. This study provides the most in-depth investigation of factors independently associated with serum GDF15 concentrations in patients with, and without, T2DM who have NAFLD, and also demonstrates that older age, higher HbA1c, higher AST, higher hs-CRP, lower e-GFR and metformin use (but not gut microbiota composition, adipokines or measures of satiety) were all independently associated with higher serum GDF-15 concentrations. Collectively, these factors explained a large proportion (60\%) of the total variance in GDF-15 concentrations. Moreover, ROC curve analysis also confirmed that $\mathrm{HbA1c}$ was a good predictor of high GDF-15 concentrations.

Our findings that a serum GDF-15 concentration of $1193.7 \mathrm{pg} /$ $\mathrm{ml}$ was the optimal threshold for predicting $\geq \mathrm{F} 3$ fibrosis are consistent with other recent work carried out in an Asian NAFLD cohort where a serum GDF-15 concentration of $1520 \mathrm{pg} / \mathrm{ml}$ was found to be the optimal threshold for predicting histologically

Table 4. Multivariable linear regression models explaining variance in serum GDF-15 concentrations.

\begin{tabular}{|c|c|c|c|}
\hline Independent variables & $R$-square $\left(R^{2}\right)$ of regression model & $R^{2}$ change & $p$-value \\
\hline Model 1 & 0.296 & 0.296 & $<0.00001$ \\
\hline \multicolumn{4}{|l|}{$\mathrm{HbA} 1 \mathrm{c}(\mathrm{mmol} / \mathrm{mol})$} \\
\hline Model 2 & 0.397 & 0.102 & $<0.001$ \\
\hline \multicolumn{4}{|c|}{$\mathrm{HbA} 1 \mathrm{c}(\mathrm{mmol} / \mathrm{mol})$ and age (yrs) } \\
\hline Model 3 & 0.480 & 0.083 & $<0.001$ \\
\hline \multicolumn{4}{|c|}{$\mathrm{HbA} 1 \mathrm{c}(\mathrm{mmol} / \mathrm{mol})$, age (yrs) and AST (IU/l) } \\
\hline Model 5 & 0.572 & 0.030 & 0.013 \\
\hline \multicolumn{4}{|c|}{$\mathrm{HbA1c}(\mathrm{mmol} / \mathrm{mol})$, age (yrs), AST (IU/l), metformin use (yes) and hs-CRP (mg/l) } \\
\hline Model 6 & 0.60 & 0.028 & 0.015 \\
\hline
\end{tabular}

Sample size, $n=99$. In all regression models, the dependent variable was the logarithmically transformed serum GDF- 15 concentrations (pg/ml). NB: $R$-square $\left(R^{2}\right.$ or the coefficient of determination) is a statistical measure in a regression model that determines the proportion of variance in the dependent variable that can be explained by the independent variables.

AST aspartate aminotransferase, hs-CRP high-sensitivity C-reactive protein, e-GFR estimated glomerular filtration rate, $C l$ confidence interval. 


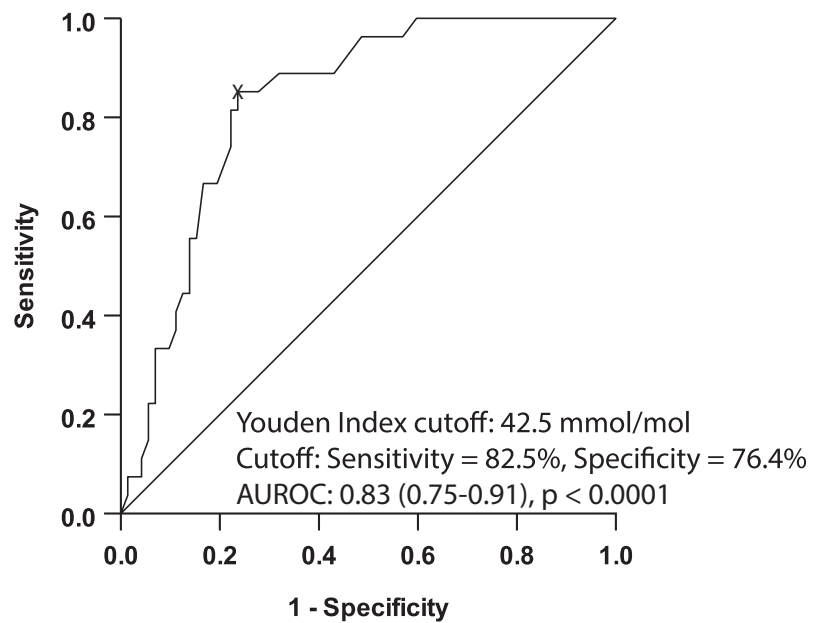

Fig. 4 Receiver-operating characteristic curve of HbA1c concentrations for a high serum GDF-15 concentration. State variable was serum GDF-15 concentrations $<1193.7 \mathrm{pg} / \mathrm{ml}$ vs. $\geq 1193.7 \mathrm{pg} / \mathrm{ml}$ ( 0 and 1 , respectively). Sample size $n=99$.

proven advanced fibrosis ( $\geq \mathrm{F} 3$ liver fibrosis) [13]. Furthermore, data from a recent large multicentre transcriptomics study identified hepatic GDF-15 expression as a key factor strongly and positively associated with liver fibrosis severity in patients with NAFLD [10]. In addition to liver fibrosis, our findings that GDF15 concentrations were significantly increased in patients with NAFLD and T2DM compared to those without T2DM, are consistent with various previous studies indicating that GDF-15 concentrations are increased in patients with T2DM [7, 26, 27].

Importantly, a strength of our study is the significant proportion of patients with T2DM and NAFLD (42.4\%) and the wide range of $\mathrm{HbA} 1 \mathrm{c}$ concentrations ( 27.0 to $95.0 \mathrm{mmol} / \mathrm{mol}$ ). Considering the chronic nature of NAFLD and that GDF-15 expression is stressinducible, it is likely that a range of factors commonly associated with T2DM and/or liver fibrosis is also associated with increasing GDF-15 concentrations. Considering this, we investigated multiple factors and found that, increased $\mathrm{HbA} 1 \mathrm{c}$ concentrations were most strongly associated with increased GDF-15 concentrations and that $\mathrm{HbA} 1 \mathrm{c}$ concentrations were a good predictor of high $(\geq 1193.7 \mathrm{pg} / \mathrm{ml})$ GDF-15 concentrations with an AUROC of 0.83 (95\% Cl 0.75-0.91). Interestingly, the optimal cut-off of $\mathrm{HbA} 1 \mathrm{c}$ concentration for predicting high GDF-15 concentrations was $42.5 \mathrm{mmol} / \mathrm{mol}$, which is remarkably similar to the threshold for diagnosing pre-diabetes in patients. In addition to this, we found that $\mathrm{HbA} 1 \mathrm{c}$ concentrations explained a large proportion $(\sim 30 \%)$ of the total variance in circulating GDF-15 and were a good predictor of high GDF-15 concentrations, supporting our findings that GDF15 concentrations may be involved in the relationship between T2DM and liver fibrosis. These findings could suggest that chronic hyperglycaemia has a role in increasing the circulating concentrations of GDF-15 in patients with both NAFLD and T2DM. Interestingly, administration of a high glucose load resulted in a rise in serum GDF-15 concentrations in both non-obese and obese individuals suggesting a potential direct role of hyperglycaemia on increased circulating GDF-15 concentrations [28, 29]. Conversely, given the increased NAFLD severity observed in patients with coexisting T2DM, it is also likely that an increased hepatic expression of GDF-15, due to hepatic inflammation and/or fibrosis and exacerbated by the presence of T2DM, also contributes to elevations in circulating concentrations of GDF-15. Furthermore, whilst a growing body of evidence suggests that GDF-15 may have a pro-fibrogenic role within the liver $[13,14]$, others have found GDF-15 to be protective and to ameliorate NASH and other metabolic disorders in mice $[30,31]$. Consequently, further work should be carried out to elucidate the functional role of GDF-15 in liver fibrosis in NAFLD. Additionally, research should look to explore the potentially additive effects of hyperglycaemia, and other factors involved in the T2DM milieu, on the expression and circulating concentrations of GDF-15.

Recent pre-clinical and clinical studies of T2DM suggest that GDF-15 expression is also increased by oral metformin treatment and that the beneficial effects of metformin on weight loss (and associated hyperglycaemia) may be mediated by metformininduced GDF-15 acting centrally to suppress appetite [18, 19]. In our study, we show for the first time that metformin treatment is associated with higher GDF-15 concentrations in patients with T2DM and NAFLD, and this association is independent of potential confounding factors. However, in contrast to $\mathrm{HbA1C}$, we found that metformin treatment explained very little of the total variance in GDF-15 concentrations within our cohort $(6 \%$ vs $~ 30 \%$ for $\mathrm{HbA1c}$ ). Similarly, we found that of the investigated inflammatory markers, only increased hs-CRP was independently associated with increased GDF-15 concentrations. However, similar to metformin treatment, hs-CRP only explained a small proportion (3\%) of the total variance in GDF-15 concentrations. Furthermore, we found that changes in the faecal microbiota, circulating LPS and adipokine concentrations and patient-reported appetite, hunger and/or satiety were not independently associated with serum GDF-15 concentrations.

Our study has some limitations. Firstly, we used the validated VCTE-derived threshold of $\geq 8.2 \mathrm{kPa}$ and $\geq 9.7 \mathrm{kPa}$ as proxies for the identification of patients with $\geq \mathrm{F} 2$ and $\geq \mathrm{F} 3$ fibrosis, respectively [23], instead of liver histology diagnosed fibrosis. That said, growing evidence indicates that liver VCTE has good diagnostic accuracy for the identification of liver fibrosis in patients with NAFLD [32]. Furthermore, a recent large study validated the use of a liver VCTE threshold of $\geq 8.2 \mathrm{kPa}$ and $\geq 9.7 \mathrm{kPa}$ as good diagnostic thresholds for identifying $\geq F 2$ (AUROC; $0.77,95 \% \mathrm{Cl} 0.72-0.82$ ) and $\geq \mathrm{F} 3$ (AUROC; $0.80,95 \%$ Cl $0.75-0.84$ ) fibrosis validated by histology [23]. Our study also utilised a relatively small cohort and further work should also be carried out in larger cohorts with access to liver biopsy data to further investigate the role of circulating GDF15 in the relationship between T2DM and liver fibrosis. Whilst evidence does suggest that GDF-15 may have a pro-fibrogenic role within the liver and we found that $\mathrm{HbA} 1 \mathrm{c}$ explains almost $30 \%$ of the variance in GDF-15 concentrations, our findings showing that GDF-15 may be involved in the known association between T2DM and liver fibrosis should be interpreted with caution. With the current study design, we are unable to address causation and we suggest that further work is required to explore the functional role of GDF-15 in the known association between T2DM and liver fibrosis in patients with NAFLD. We also did not collect data on metformin treatment dosage or duration of treatment within the current cohort and we are not able to investigate whether dose-or time-dependent effects exist between metformin use and serum GDF-15 concentrations.

In conclusion, in patients with NAFLD and T2DM, GDF-15 concentrations predicted both $\geq \mathrm{F} 2$ and $\geq F 3$ liver VCTE determined fibrosis, and GDF-15 concentrations may be involved in the association between T2DM and liver fibrosis in NAFLD. Furthermore, we explained a large proportion $(\sim 60 \%)$ of the variance in GDF-15 concentrations and found that $\mathrm{HbA} 1 \mathrm{c}$ alone explained almost $30 \%$ of that variance. Further investigations are warranted to establish the causal or consequential role of GDF-15 in liver fibrosis and to further explore the potential implementation of circulating GDF-15 as a biomarker for liver fibrosis in patients with NAFLD and T2DM.

\section{REFERENCES}

1. Byrne CD, Targher G. NAFLD: a multisystem disease. J Hepatol. 2015;62:S47-64. 
2. Byrne CD, Targher G. NAFLD as a driver of chronic kidney disease. J Hepatol. 2020;72:785-801.

3. Bril F, Cusi K. Management of nonalcoholic fatty liver disease in patients with type 2 diabetes: a call to action. Diabetes Care. 2017;40:419-30.

4. Targher G, Lonardo A, Byrne CD. Nonalcoholic fatty liver disease and chronic vascular complications of diabetes mellitus. Nat Rev Endocrinol. 2018;14:99-114.

5. Wischhusen J, Melero I, Fridman WH. Growth/differentiation factor-15 (GDF-15): from biomarker to novel targetable immune checkpoint. Front Immunol.2020;11:951.

6. Dostálová I, Roubícek T, Bártlová M, Mráz M, Lacinová Z, Haluzíková $D$, et al. Increased serum concentrations of macrophage inhibitory cytokine-1 in patients with obesity and type 2 diabetes mellitus: the influence of very low calorie diet. Eur J Endocrinol. 2009;161:397-404.

7. Kempf T, Guba-Quint A, Torgerson J, Magnone MC, Haefliger C, Bobadilla M, et al. Growth differentiation factor 15 predicts future insulin resistance and impaired glucose control in obese nondiabetic individuals: results from the XENDOS trial. Eur J Endocrinol. 2012;167:671-8.

8. Sarkar S, Legere S, Haidl I, Marshall J, MacLeod JB, Aguiar C, et al. Serum GDF15, a promising biomarker in obese patients undergoing heart surgery. Front Cardiovasc Med. 2020;7:103.

9. Lee ES, Kim SH, Kim HJ, Kim KH, Lee BS, Ku BJ. Growth differentiation factor 15 predicts chronic liver disease severity. Gut Liver. 2017;11:276-82.

10. Govaere O, Cockell S, Tiniakos D, Queen R, Younes R, Vacca M, et al. Transcriptomic profiling across the nonalcoholic fatty liver disease spectrum reveals gene signatures for steatohepatitis and fibrosis. Sci Transl Med. 2020;12: eaba4448.

11. Wang J, Wei L, Yang $X$, Zhong J. Roles of growth differentiation factor 15 in atherosclerosis and coronary artery disease. J Am Heart Assoc. 2019;8:e012826.

12. Nair V, Robinson-Cohen $C$, Smith MR, Bellovich KA, Bhat ZY, Bobadilla M, et al. Growth differentiation factor-15 and risk of CKD progression. J Am Soc Nephrol. 2017;28:2233-40.

13. Koo BK, Um SH, Seo DS, Joo SK, Bae JM, Park JH, et al. Growth differentiation factor 15 predicts advanced fibrosis in biopsy-proven non-alcoholic fatty liver disease. Liver Int. 2018;38:695-705.

14. Qi P, Ma MZ, Kuai JH. Identification of growth differentiation factor 15 as a profibrotic factor in mouse liver fibrosis progression. Int J Exp Pathol. 2021;102:148-56.

15. Takenouchi Y, Kitakaze K, Tsuboi K, Okamoto Y. Growth differentiation factor 15 facilitates lung fibrosis by activating macrophages and fibroblasts. Exp Cell Res. 2020;391:112010.

16. Adolph TE, Grander C, Grabherr F, Tilg H. Adipokines and non-alcoholic fatty liver disease: multiple interactions. Int J Mol Sci. 2017;18:1649.

17. Oh TG, et al. A universal gut-micrÿobiome-derived signature predicts cirrhosis. Cell Metab. 2020;32:901.

18. Coll AP, Chen M, Taskar P, Rimmington D, Patel S, Tadross JA, et al. GDF15 mediates the effects of metformin on body weight and energy balance. Nature. 2020;578:444-8.

19. Day EA, Ford RJ, Smith BK, Mohammadi-Shemirani P, Morrow MR, Gutgesell RM, et al. Metformin-induced increases in GDF15 are important for suppressing appetite and promoting weight loss. Nat Metab. 2019;1:1202-8.

20. Scorletti E, Afolabi PR, Miles EA, Smith DE, Almehmadi A, Alshathry A, et al. Synbiotics alter fecal microbiomes, but not liver fat or fibrosis, in a randomized trial of patients with nonalcoholic fatty liver disease. Gastroenterology. 2020;158:1597-1610.e7.

21. Scorletti E, Afolabi PR, Miles EA, Smith DE, Almehmadi A, Alshathry A, et al. Design and rationale of the INSYTE study: a randomised, placebo controlled study to test the efficacy of a synbiotic on liver fat, disease biomarkers and intestinal microbiota in non-alcoholic fatty liver disease. Contemp Clin Trials. 2018;71:113-23.

22. Florkowski CM, Chew-Harris JS. Methods of estimating GFR - different equations including CKD-EPI. Clin Biochem Rev. 2011;32:75-9.

23. Eddowes PJ, Sasso M, Allison M, Tsochatzis E, Anstee QM, Sheridan D, et al. Accuracy of fibroscan controlled attenuation parameter and liver stiffness measurement in assessing steatosis and fibrosis in patients with nonalcoholic fatty liver disease. Gastroenterology. 2019;156:1717-30.

24. Thapa BR, Walia A. Liver function tests and their interpretation. Indian J Pediatr. 2007;74:663-71.

25. Preacher, K, Leonardelli, G. Calculation for the Sobel test. An interactive calculation tool for mediation tests. 2010. http://quantpsy.org/sobel/sobel.htm. Accessed 3 June 2021.

26. Bao X, Borné Y, Muhammad IF, Nilsson J, Lind L, Melander O, et al. Growth differentiation factor 15 is positively associated with incidence of diabetes mellitus: the Malmö Diet and Cancer-Cardiovascular Cohort. Diabetologia. 2019;62:78-86.

27. Adela R, Banerjee SK. GDF-15 as a target and biomarker for diabetes and cardiovascular diseases: a translational prospective. J Diabetes Res. 2015;2015:490842.
28. Schernthaner-Reiter MH, Kasses D, Tugendsam C, Riedl M, Peric S, Prager G, et al. Growth differentiation factor 15 increases following oral glucose ingestion: effect of meal composition and obesity. Eur J Endocrinol. 2016;175:623-31.

29. Karczewska-Kupczewska M, Kowalska I, Nikolajuk A, Adamska A, Otziomek E, Gorska $M$, et al. Hyperinsulinemia acutely increases serum macrophage inhibitory cytokine-1 concentration in anorexia nervosa and obesity. Clin Endocrinol. 2012;76:46-50.

30. Kim KH, Kim SH, Han DH, Jo YS, Lee YH, Lee MS. Growth differentiation factor 15 ameliorates nonalcoholic steatohepatitis and related metabolic disorders in mice. Sci Rep. 2018;8:6789.

31. Chrysovergis K, Wang X, Kosak J, Lee SH, Kim JS, Foley JF, et al. NAG-1/GDF-15 prevents obesity by increasing thermogenesis, lipolysis and oxidative metabolism. Int J Obes. 2014;38:1555-64.

32. Selvaraj EA, et al. Diagnostic accuracy of elastography and magnetic resonance imaging in patients with NAFLD: a systematic review and meta-analysis. J Hepatol. 2021. https://doi.org/10.1016/j.jhep.2021.04.044.

\section{ACKNOWLEDGEMENTS}

We would like to thank the National Institute for Health Research Southampton Biomedical Research Centre for funding this study and supporting the authors throughout the study. We also thank Debbie Smith, Sanchia Triggs, Gemma Rood and Jennifer Hedges without whose help this study would not have been possible and Lucinda England for research governance administration. Finally, we thank all the study volunteers for their invaluable contribution towards furthering knowledge about NAFLD. The datasets generated during and/or analysed during the current study are available from the corresponding author on reasonable request.

\section{AUTHOR CONTRIBUTIONS}

JB, BSc (Hons), MRes, PhD candidate (formal analysis: lead; writing-original draft: lead; writing - review and editing: lead; data curation: supporting). ES, MD, PhD (formal analysis: supporting, data curation: supporting; investigation: supporting). LBB, PhD (formal analysis: supporting; data curation: supporting). PRA, PhD (formal analysis: supporting). GT, MD (writing-original draft: supporting; writing-review and editing: supporting). PCC, BSC (Hons), PhD, DPhil, RNutr, FRSB, FAfN (conceptualisation: supporting; funding acquisition: supporting, supervision: supporting; validation: supporting; writing - original draft: supporting; writing-review and editing: supporting). JKS, BSC, DPhil, FRSB (conceptualisation: supporting; funding acquisition: lead; supervision: lead, investigation: supporting; writing—original draft: supporting; writing-review and editing: supporting). CCB, PhD, FRCPath, FRCP (conceptualisation: lead; funding acquisition: lead; supervision: supporting; validation: supporting; visualisation: supporting; writing—original draft: supporting; writingreview and editing: supporting).

\section{FUNDING}

JB, PRA, PCC, JKS and CDB are supported by the National Institute for Health Research through the NIHR Southampton Biomedical Research Centre (Grant Number IS-BRC20004). This research was funded in part by the Wellcome Trust [Grant number $206453 / Z / 17 / Z$ to JKS]. For the purpose of open access, the author has applied a CC BY public copyright license to any Author Accepted Manuscript version arising from this submission.

\section{COMPETING INTERESTS}

The authors declare no competing interests.

\section{ADDITIONAL INFORMATION}

Supplementary information The online version contains supplementary material available at https://doi.org/10.1038/s41387-021-00170-3.

Correspondence and requests for materials should be addressed to Jaswinder $\mathrm{K}$. Sethi or Christopher D. Byrne.

Reprints and permission information is available at http://www.nature.com/ reprints

Publisher's note Springer Nature remains neutral with regard to jurisdictional claims in published maps and institutional affiliations. 
Open Access This article is licensed under a Creative Commons (c) Attribution 4.0 International License, which permits use, sharing,
adaptation, distribution and reproduction in any medium or format, as long as you give appropriate credit to the original author(s) and the source, provide a link to the Creative Commons license, and indicate if changes were made. The images or other third party material in this article are included in the article's Creative Commons license, unless indicated otherwise in a credit line to the material. If material is not included in the article's Creative Commons license and your intended use is not permitted by statutory regulation or exceeds the permitted use, you will need to obtain permission directly from the copyright holder. To view a copy of this license, visit http://creativecommons. org/licenses/by/4.0/.

(๑) The Author(s) 2021 